\title{
Immunohistochemical study on roe deer haemal nodes
}

\author{
Y. Akaydın Bozkurt ${ }^{1}$, E. Karadağ Sarı ${ }^{2}$, M. Kabak ${ }^{3}$ \\ ${ }^{1}$ Department of Histology and Embryology, Faculty of Veterinary Medicine, Mustafa Kemal University, Hatay, Turkey \\ 2Department of Histology and Embryology, Faculty of Veterinary Medicine, Kafkas University, Kars, Turkey \\ ${ }^{3}$ Department of Anatomy, Faculty of Veterinary Medicine, Ondokuz Mayıs University, Samsun, Turkey
}

[Received: 26 April 2017; Accepted: 5 October 2017]

Background: The aim of this study was to investigate the immunohistochemical characteristics of the haemal nodes located in the abdominal and thoracic cavities in roe deer (Capreolus capreolus).

Materials and methods: In this study, 2 adult male and 2 adult female roe deers in addition to 2 roe deer foetuses at the late foetal stages were used. The avidin-biotin-peroxidase technique was applied to anti-CD3, anti-CD79 $\alpha C y$, anti-macrophage, anti-S100 primary antibodies.

Results: Positive results were gained for all the antibodies used. Many CD3 positive T-lymphocytes were seen in the lymphoid tissue areas of the foetal haemal nodes, in the germinal centre of the lymph follicles of the adult haemal nodes and in their lymphatic cords. CD79acy positive cells were less in number while S-100 protein was positive in both lymph follicles and in sinuses of the adult haemal nodes. In foetal haemal nodes, cells positive for S-100 protein were more prevalent in the sinuses. For the MAC387 primary antibody, there were positive macrophages seen in the capsule and trabeculae of the haemal nodes, located denser in males. Macrophage cells in the lymphoid structures and granulocytes in the sinuses of the haemal nodes of both adults and foetuses were positive for MAC387.

Conclusions: This study is the first one to define the detailed structural features immunohistochemically in the haemal nodes of the roe deer. Even it's concluded that the results were similar with the other ruminants, it's also pointed out that there are certain differences among the foetal, adult male and female haemal nodes of the roe deer. (Folia Morphol 2018; 77, 2: 266-271)

Key words: CD3, CD79 $\alpha$ cy, macrophage, S-100 protein, immunohistochemistry, haemal node, roe deer

\section{INTRODUCTION}

Haemal nodes are commonly found in the dorsal parts of the abdominal and thoracic cavities, defined in ruminants, and are common throughout the vena cava and abdominal aorta in the sub lumbar region $[9,10]$. The number, size, and histological characteristics of the haemal nodes observed throughout the blood vessels may vary within wide limits $[2,10]$. Haemal nodes are lymphoid organs that have a function similar to that of the spleen and lymph node, and function in the immunological defence of the body in blood storage and filtration [8].

Haemal nodes develop during foetal life from lymph node primordia that lose their lymph vessels. 
Table 1. Antibody specificity, dilution rates and incubation times

\begin{tabular}{|c|c|c|c|c|}
\hline Specificity & $\mathrm{pAb} / \mathrm{mAb}$ (clone) ${ }^{*}$ & Reactivity & Source & Dilution \\
\hline CD3 & $\mathrm{mAb}(\mathrm{SP} 7)$ & T lymphocyte & Abcam & 1/100 (overnight $/ 4^{\circ} \mathrm{C}$ ) \\
\hline CD79 $\alpha$ cy & mAb (HM57) & B lymphocyte & Dako & $1 / 25$ \\
\hline L1 & mAb (MAC387) & Granulocyte, monocyte, macrophage & Abcam & 1/100 (15 min/room temperature) \\
\hline S100 & $\mathrm{pAb}$ & Interdigitating reticulum cells & Abcam & 1/100 (2 h/room temperature) \\
\hline
\end{tabular}

${ }^{*} \mathrm{mAb}$ - monoclonal antibody; pAb — polyclonal antibody

Therefore, haemal nodes receive all their cells and antigens from the blood. Haemal nodes are thought to be responsive to blood-borne antigens. In healthy adults, many follicles may form as a result of antigenic stimulation. Sinuses are wide, with a small amount of macrophages and lymphocytes. Diffuse lymphatic tissue contains relatively fewer lymphocytes, but macrophages that digest erythrocytes and granulocytes are present in large quantities [9].

There are various studies on morphology, histochemistry and immmunohistochemistry of haemal nodes in ruminants $[5,8,11,12,19]$. Morphology of haemal nodes in the roe deer (Capreolus capreolus) has been studied by Akaydın Bozkurt and Kabak [1], but there is no study available on immmunohistochemistry of haemal nodes in the roe deer.

The objective of the study is to identify immunohistochemical (IHC) characteristics and determine the distribution of T-lymphocytes, B-lymphocytes, macrophages and follicular dendritic cells immunohistochemically, by revealing the differences between haemal nodes of foetal, adult male and adult female roe deer as well.

\section{MATERIALS AND METHODS}

The haemal nodes employed as research material in the study was belonging to 2 adult males and 2 adult females which had been injured in the Samsun region due to firearms and traffic accidents and brought to Ondokuz Mayis University, Veterinary Faculty Clinics for treatment but failed to survive. These animals examined and were not found to have any signs of infectious or parasitic disease. It was noticed that one of the female roe deer was pregnant. Haemal nodes of these 2 row deer foetuses which have developed and been in the late foetal period were also used as research material. The haemal nodes harvested from the thoracic and abdominal cavities were fixed with a $10 \%$ neutral formol for $24 \mathrm{~h}$. Fol- lowing the routine histological procedure, standard avidin-biotin-peroxidase technique (ABC) was applied to 4-5 $\mu \mathrm{m}$ serial tissue sections taken on adhesive glass slides. Sections were passed through xylol and alcohol solutions. Heat-mediated antigen retrieval with citrate buffer ( $\mathrm{pH}: 6)$, was performed before proceeding with the IHC staining protocol. Endogenous peroxidase activity was blocked by quenching with $3 \%$ hydrogen peroxide for $10 \mathrm{~min}$. The IHC staining protocol employed was an UltraVision ${ }^{\text {TM }}$ Large Volume Detection System (Ultra V Block, Biotinylated Goat Anti-Polyvalent, Streptavidin Peroxidase; Lab Vision Corp., Thermo Fisher Scientific Inc., Fremont, CA). After application of Ultra $\mathrm{V}$ Block Nonspecific Blocking Reagent (10 min), primary antibody solutions were dropped on the sections and incubation was allowed (Table 1). After application of biotinylated goat anti-polyvalent (15 $\mathrm{min}$ ) and streptavidin peroxidase (15 min), AEC chromogen (Labvision) for CD3, anti-macrophage and anti-S100 for $10 \mathrm{~min}, \mathrm{DAB}$ chromogen for CD79 $\alpha$ cy primary antibody were used so that the antigen-antibody reaction can be made visible. Mayer's haematoxylin was applied for $1 \mathrm{~min}$ for the background staining. Phosphate buffer saline (PBS; $0.01 \mathrm{M}, \mathrm{pH}$ 7.4) were employed in all washing processes. PBS was used instead of the primary antibodies for negative controls. Evaluation of the results was carried out by examining the sections on the Olympus BX50 model research microscope, and pictures of selected areas were taken and used in the study (Table 1).

\section{RESULTS}

Great deal of CD3 positive T-lymphocytes were observed in lymphatic cords and interfollicular regions of haemal nodes of adults. CD3 positive T-lymphocytes were observed in the germinal centres of the lymph follicles and in lesser amounts in the mantle zones (Fig. 1A, B). A large amount of CD3 positive 


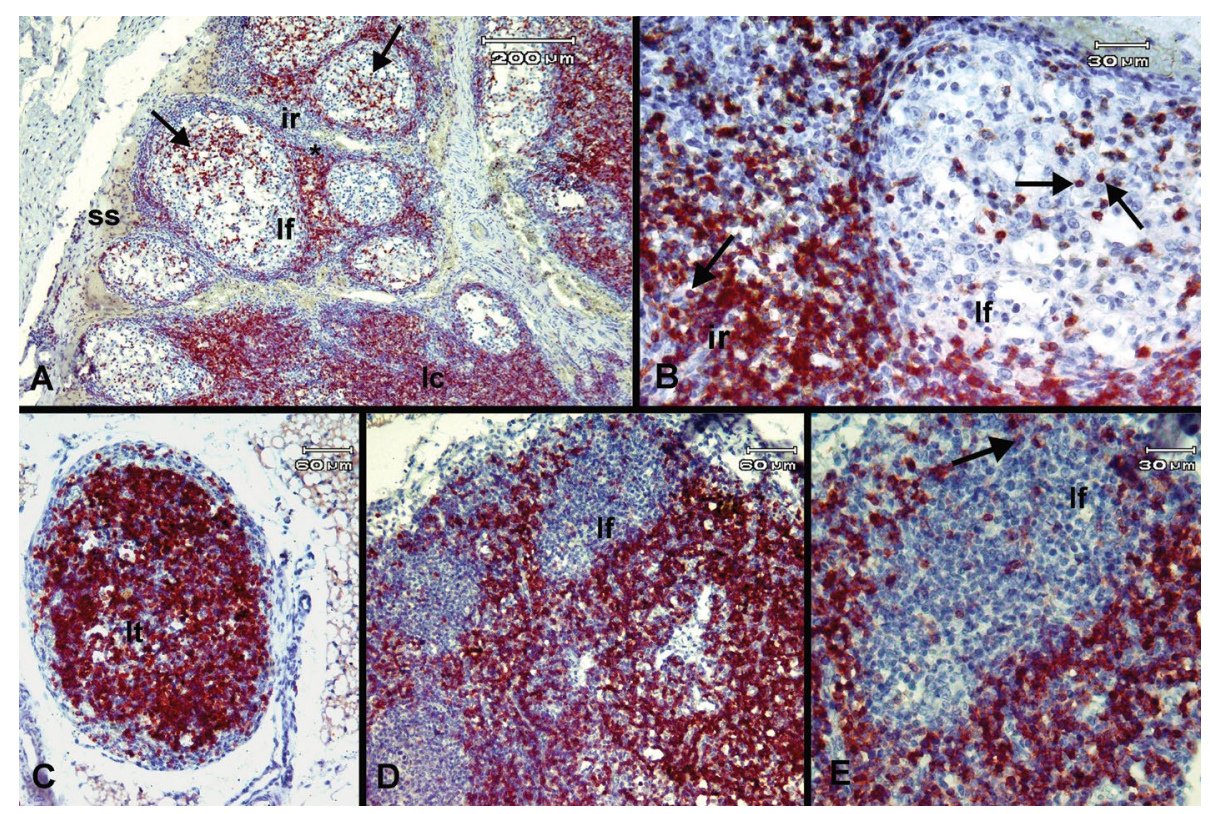

Figure 1. CD3 positive T-lymphocytes (arrows) in the haemal node (A, B) of adult female roe deer and in the haemal node (C, D, E) of the foetal roe deer; ir — interfollicular region; If — lymph follicle; It — lymphoid tissue; ss — subcapsular sinus.
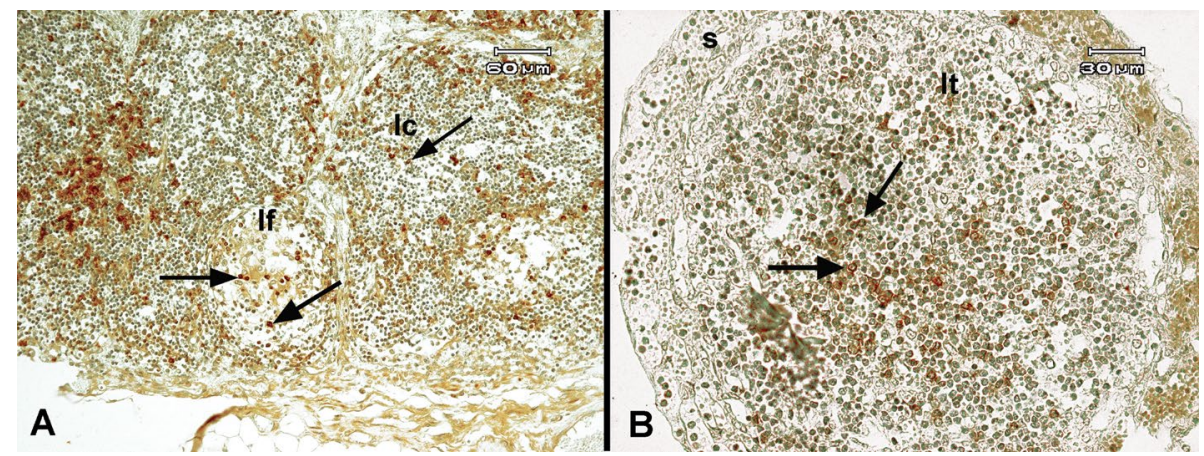

Figure 2. CD79 $\alpha$ cy positive B-lymphocytes (arrows) in the haemal node (A) of adult female roe deer and in the haemal node (B) of the foetal roe deer; Ic — lymphatic cord; If — lymph follicle; It — lymphoid tissue; $\mathrm{s}$ - sinus.

T-lymphocytes were observed in the lymphoid tissue of foetal haemal node (Fig. 1C). There were also sporadic positive cells available in the lymph follicles of very few foetal haemal nodes, where only a few primary lymphoid follicles were formed (Fig. 1D, E). It has been observed that the sinuses of the haemal nodes of both adults and foetuses contain CD3-positive T-lymphocytes (Fig. 1A-E).

In the haemal nodes of female and male there were fewer CD79 $\alpha$ cy positive cells in the germinal centres of the lymph follicles and much fewer of it in the interfollicular zone and lymphatic cords (Fig. 2A). There were more positive cells available in the haemal node of female than in the haemal node of male. It was observed that the cells that gave strong positive reaction in the female were usually plasma cells. It was determined that there were those cells that gave positive reaction to $C D 79 \alpha c y$ in the lymphoid tissue of the foetal haemal nodes (Fig. 2B).

It was observed that MAC387 positive cells were found abundantly, in particular, in sub capsular sinus and trabecular sinus in adults. However, positive cells were also found in trabeculae and in lymphatic cords (Fig. 3A, B). There were more MAC387 positive cells observed in the haemal nodes of male than in that of female. Only a few (rarely) positive cells in the sub capsular sinus were found while positive cells were found in the lymphoid tissues of the foetal haemal 


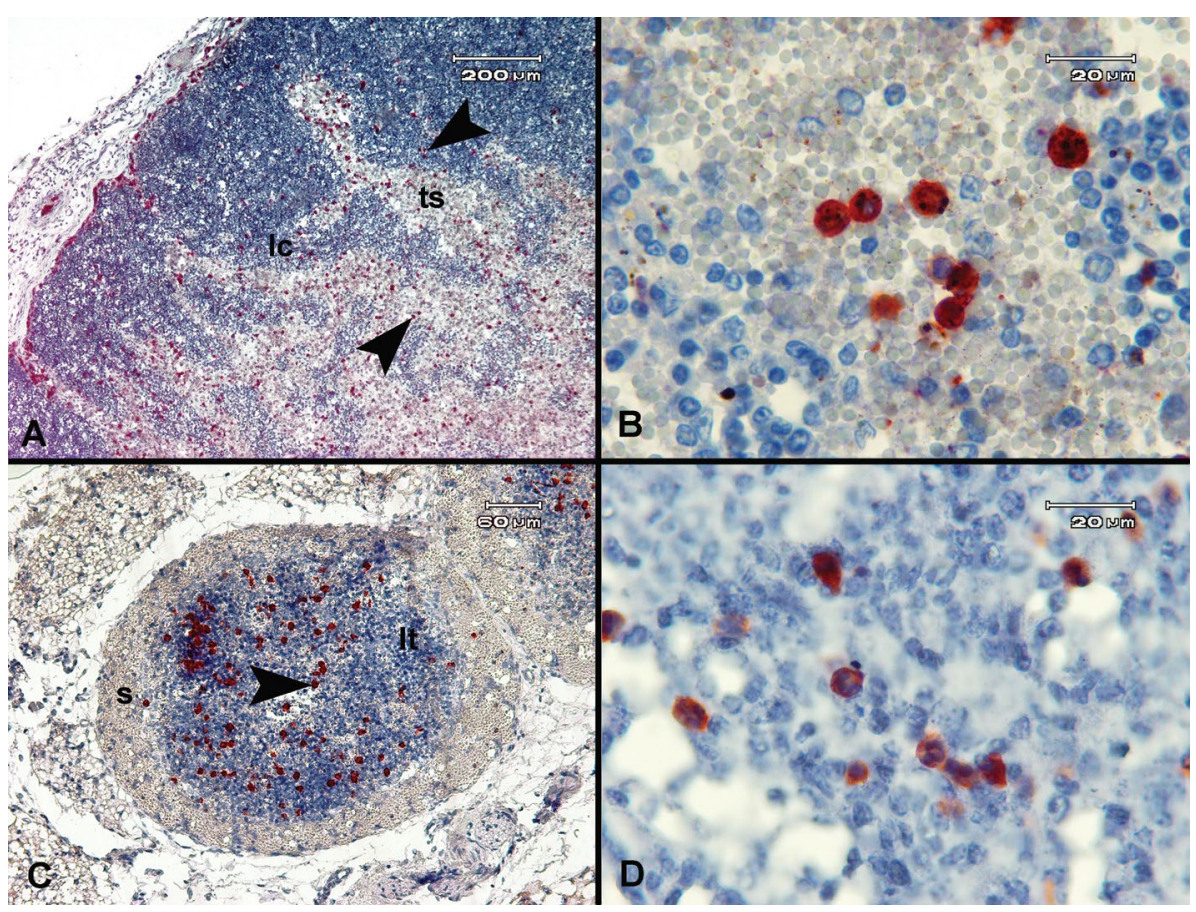

Figure 3. MAC387 positive macrophages and granulocytes in the haemal node (A, B) of adult male roe deer and in the haemal node (C, D) of the foetal roe deer; Ic — lymphatic cord; It — lymphoid tissue; s - sinus; ts - trabecular sinus; arrowheads — MAC387 positive cells.

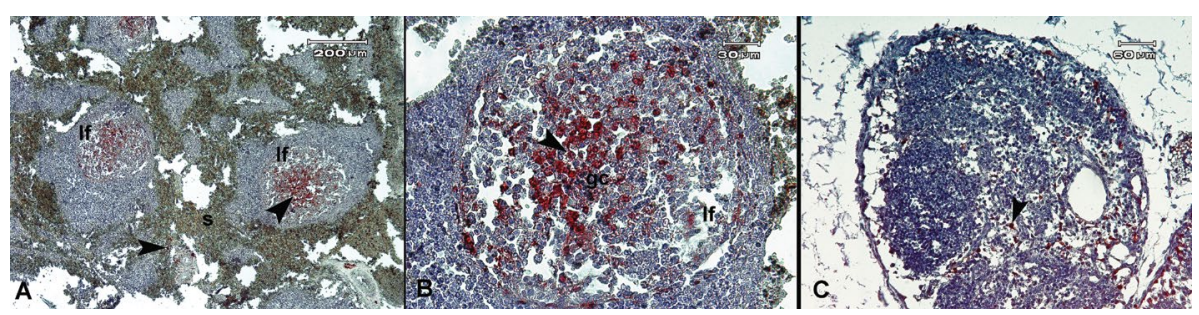

Figure 4. S100 positive cells (arrowheads) in the haemal node (A, B) of adult male roe deer and in the haemal node (C) of the foetal roe deer; gc - germinal centre; If — lymph follicle; $s$ - sinus.

nodes (Fig. 3C, D). There were positive cells also in the small sinuses located inside the lymphoid tissue of some foetal haemal nodes. Most of the cells showing positivity for MAC387 were granulocytes in the foetal haemal node.

S100 positive cells have been observed in both lymph follicles and sinuses of haemal nodes of adults (Fig. 4A, B). No positive reaction cells were found in the lymphatic cords while there were abundant follicular dendritic cells stained with $\mathrm{S} 100$ positive in the germinal centres of lymph follicles. There were cells showing positivity in the very few haemal nodes, mostly in the sinuses, although there were no cells showed positive staining for $\mathrm{S} 100$ in the foetal haemal nodes (Fig. 4C).

\section{DISCUSSION AND CONCLUSIONS}

There is no study that examines the haemal nodes in the roe deer with IHC methods when the literature related to haemal nodes is browsed. It is seen in the studies with regard to haemal node, where IHC methods were employed, that monoclonal mouse for T-lymphocytes, anti-human $B$ primary antibodies for anti-human $\mathrm{T}$ - and B-lymphocytes were used in the frozen sections of haemal nodes in the Korean native goat [18]; and CD4, CD8, T19, CD45R, MHC class I and MHC class II monoclonal antibody (mAbs) were used for the characterisation and distribution of lymphocyte subgroups in the frozen sections of the haemal nodes of the sheep [16]. Guerrero et al. [11] have showed CD3 positive T-lymphocytes using polyclonal anti-CD3 primary antibody in paraffin 
sections of the haemal nodes in the lberian red deer. Zidan and Pabst [19] have performed IHC study of the haemal nodes of the dromedary camel using CD3 (rabbit anti-human), CD22 (mouse anti-bovine) and MHC class II/DR (mouse anti-bovine) mAbs in the frozen sections. Attempts have been made to reveal the existence and distribution of, in particular, T- and B-lymphocytes in either frozen or in paraffin sections in the ruminant species even with different primary antibodies when all such studies are taken into consideration.

Ceccarelli et al. [4] have observed that most of the lymphoid cells in the haemal nodes and in the interfollicular cords of the sheep and cows and the cells in the germinal centres of some secondary follicles could be identified as T-lymphocytes and most of the cells in the primary follicles and in the cortex of the secondary follicles could be identified as B-lymphocytes. Yoon et al. [18] have reported that B-lymphocytes were mainly located in lymphoid follicles of the haemal node, and that they were sparsely scattered in the sub capsular sinus. In the same study, it is mentioned that T-lymphocytes were sparsely scattered in the diffuse lymphatic tissues of the haemal node. Zidan and Pabst [19] reported that CD3-positive T lymphocytes were localised mainly at the margin of lymphoid follicles and in the medullary cords and that CD22-positive B lymphocytes were predominantly found in lymphoid follicles which are the typical localisation areas of these cells. Casteleyn et al. [3] reported that T cell lymphocytes marked with CD3 in the haemal node of bovine were mostly localised at the paracortex and the interfolic zone, and that medullary cords also contained CD3-positive cells, and that there were very few CD3-positive cells observed in the mantle zone of the follicles but almost no positive cells were observed in the germinal centre. They have determined that CD21-marked B-lymphocytes were confined to the follicles and had a predominant position in the germinal centres. Guerrero et al. [11] suggested that lymphatic tissue capable of forming into a cord-like shape in the cervical haemal nodes was composed of plasma cells, macrophages, B- and T-lymphocytes and demonstrated the anti-CD3 positive lymphocytes in the paracortex. Distribution of T-lymphocytes appears to be more compatible with the findings of Casteleyn et al. [3] in the haemal node of bovine even though it bears a resemblance to that of sheep, goat, cattle, camel and deer in general when data related to the distribution of T-lymphocytes in the literature are examined. However, the existence of more T-lymphocyte in the germinal centres of the lymphoid follicles compared to the mantle zone differs from the findings of Casteleyn et al. [3]. Furthermore, the literature reveals no such evidence that T-lymphocytes were observed in the sinuses. It is one of the different findings in this study that T-lymphocytes were observed in the sinuses, haemal nodes of both foetuses and adult roe deer.

Whereas the existence of the B-lymphocytes in the haemal nodes has been reported and observed only in the lymph follicles by Zidan and Pabst [19] and Casteleyn et al. [3], and in the sub capsular sinus in addition to the follicles by Yoon et al. [18], this study revealed that the distribution of B-lymphocytes in the haemal nodes of roe deer was different based on the fact that B-lymphocytes in the haemal nodes of the roe deer were also observed in the interfollicular zone and medullary cords in addition to the follicles. Guerrero et al. [11] has mentioned the presence of B-lymphocytes in cord-like lymphoid tissue in deer, however, did not indicate by which method the presence of B-lymphocytes was detected in their study, and there was no photograph of B-lymphocytes in that study.

Guerrero et al. [11] reported that the lymphatic tissue capable of forming into a cord-like shape in the Iberian red deer was composed of plasma cells, macrophages, B-and T-lymphocytes, but there is no other explanation for macrophages in the study. Thorp et al. [16] reported that the macrophages containing hemosiderin reacted positively with Perl's stain, and stated that very few Perl's positive cells were found and they were located on the wall of sinuses. It was reported that lymph follicles and lymphoid cords in the haemal node of hair goat contained a lot of lymphocytes, mast cells, plasma cells, monocytes, neutrophilic granulocytes and macrophages, including eosinophilic granulocytes [12], and that lymph follicles and lymphoid cords in the haemal node of Akkaraman sheep contain macrophages [15]. Ezeasor and Singh [8] has detected the presence of macrophages in the sub capsular sinus of the haemal nodes of 1-2-month-old goats and reported that haemal nodes of 3-4-month-old goat exhibited a similar cellular composition with that of 1-month-old goat. The literature, when examined, reveals that the presence and distribution of macrophages in the haemal nodes are not studied by IHC methods. Some of the studies mention only those macrophages present in the sinuses and some of them mention only their presence in the lymphoid tissue. It is a noteworthy difference to observe more intense positive cells in 
adults, especially in sub capsular sinus and trabecular sinus than in lymphatic tissue though the findings obtained in the study were basically consistent with the findings in the literature.

Windqvist [17], who has investigated the development of haemal node in cows, reported the presence of reticular macrophages containing lipofuscin granules in the haemal nodes, in the sinuses and in the lymphatic tissue of newborn calves while not mentioning macrophages in haemal nodes in the embryonic and foetal period. The presence of MAC387 positive cells differs from the literature knowledge even though granulocytes constitute the majority in the haemal nodes of the foetal roe deer.

It was detected that IHC studies were carried out in the lymph node of rat with $\mathrm{S100}$ protein [6], RomeroPalomo et al. [13] has employed polyclonal rabbit anti-S100 primary antibody as dendritic cell marker in the lymph node of cattle. There is also a comparative study on S100 protein-immunoreactive cells in the lymph nodes of guinea pig, dog, cat, horse, pig, goat and cow [14]. There is no study available in the haemal nodes of the roe deer for follicular dendritic cells when the literature is browsed in this respect. In addition to that, there is no IHC studies carried out in the haemal nodes of healthy animals for follicular dendritic cells when the literature is browsed for other ruminants. The only study found was the study carried out on scrapie sheep [7]. For this reason, the findings obtained for $\mathrm{S} 100$ were not compared with those included in any literature.

It is not unusual to see that frozen sections are employed frequently in the IHC studies. The study carried out shows that a clear result can be obtained in paraffin sections as well. The obtained IHC findings indicate that the haemal nodes in the roe deer are immunologically active organs. It is clearly seen that there are as much differences as similarities in the distribution of these cells when compared with the other ruminant species. The work fulfilled has a feature of being the first study in terms of examining haemal node in the roe deer with IHC methods in such a way as to reveal the differences between the haemal nodes of foetal, adult male and adult female roe deer as well.

\section{REFERENCES}

1. Akaydin Bozkurt Y, Kabak M. Morphology of haemal nodes in the roe deer (Capreolus capreolus). Anat Histol Embryol. 2010; 39(5):
456-461, doi: 10.1111/j.1439-0264.2010.01016.x, indexed in Pubmed: 20624158.

2. Bacha WJ, Bacha LM. Color Atlas of Veterinary Histology. Third ed. Wiley-Blackwell, A John Wiley\&Sons, Inc, Publication, UK. 2012.

3. Casteleyn CR, Breugelmans S, Simoens $P$, et al. Morphological and immunological characteristics of the bovine temporal lymph node and hemal node. Vet Immunol Immunopathol. 2008; 126(3-4): 339-350, doi: 10.1016/j.vetimm.2008.09.010, indexed in Pubmed: 18952301.

4. Ceccarelli P, Gargiulo AM, Fagioli O, et al. Cytochemical identification of lymphocytes and other mononuclear cells in ovine and bovine hemal nodes. Comp Immunol Microbiol Infect Dis. 1986; 9(4): 297-302, indexed in Pubmed: 3026721.

5. Cerutti P, Marcaccini A, Guerrero F. A Scanning and Immunohistochemical Study in Bovine Haemal Node. Anat Histol Embryol. 1998; 27(6): 387-392, doi: 10.1111/j.1439-0264.1998. tb00212.x.

6. Cocchia D, Tiberio G, Santarelli R, et al. S-100 protein in "follicular dendritic" cells or rat lymphoid organs. An immunochemical and immunocytochemical study. Cell Tissue Res. 1983; 230(1): 95-103, indexed in Pubmed: 6342797.

7. Dassanayake RP, Truscott TC, Özyiğit MÖ, et al. Accumulation profiles of $\operatorname{Pr} P(\mathrm{Sc})$ in hemal nodes of naturally and experimentally scrapie-infected sheep. BMC Vet Res. 2013; 9: 82, doi: 10.1186/17466148-9-82, indexed in Pubmed: 23601183.

8. Ezeasor DN, Singh A, Sims DE, et al. Histology of the caprine hemal node. Acta Anat (Basel). 1988; 133(1): 16-23, indexed in Pubmed: 3213400.

9. Eurell JA, Frappier BL. Dellmann Textbook of Veterinary Histology. Sixth ed. Blackwell Publishing, USA. 2006.

10. Frandson RD, Wilke WL, Fails AD. Anatomy and Physiology of Farm Animals. Seventh ed. Wiley-Blackwell, A John Wiley\&Sons, Inc, Publication, USA. 2009.

11. Guerrero F, Cerutti $P$, Marcaccini A, et al. Histological and immunohistochemical study on Iberian red deer (Cervus elaphus hispanicus) haemal nodes. Microsc Microanal. 2012; 18(Suppl 5): 1-2, doi: 10.1017/S1431927612012664.

12. Ozaydin T, Sur E, Celik I, et al. Histological and enzyme histochemical investigation of the hemal nodes of the hair goat. Biotech Histochem. 2012; 87(6): 377-384, doi: 10.3109/10520295.2012. 652174, indexed in Pubmed: 22235874.

13. Romero-Palomo F, Risalde MA, Molina V, et al. Immunohistochemical detection of dendritic cell markers in cattle. Vet Pathol. 2013; 50(6): 1099-1108, doi: 10.1177/0300985813482951, indexed in Pubmed: 23528943.

14. Sugimura $M$, Shirogane $D$, Atoji $Y$, et al. A comparative study on S-100 protein-immunoreactive cells in lymph nodes. Nihon Juigaku Zasshi. 1990; 52(5): 1015-1021, indexed in Pubmed: 2280486.

15. Sur E, Aydın MF, Çelik IA. Light microscopic investigation on the histology and Alpha-Naphthyl Acetate Esterase (ANAE)-positive lymphocyte localization in the hemal nodes of Akkaraman sheep. Eurasian J Vet Sci. 2005; 21(1-2): 101-108.

16. Thorp BH, Seneque $S$, Staute $K$, et al. Characterization and distribution of lymphocyte subsets in sheep hemal nodes. Dev Comp Immunol. 1991; 15(4): 393-400, indexed in Pubmed: 1773862.

17. Windqvist $G$. The bovine hemal nodes. Acta Anat. 1954; 22: 108-112.

18. Yoon, YS, Lee JS, Lee HS, Kim JS. Morphological studies on the hemal node and hemolymph node in the Korean native goat. Korean J Anat. 1989; 22: 261-278.

19. Zidan M, Pabst R. Histological, histochemical and immunohistochemical study of the haemal nodes of the dromedary camel. Anat Histol Embryol. 2004; 33(5): 284-289, doi: 10.1111/j.14390264.2004.00550.x, indexed in Pubmed: 15352881. 